\title{
Synthesis of Spiro Pyrrolidines From the Addition of Schiff Bases to $\alpha$-Arylidene Cyclohexanones
}
A.J. Al-Hamdany
A.M. Mustafa
M.A. Hamza
Dept. of Chemistry
College of Science
Dept. of Chemistry
College of Education
University of Mosul

\section{University of Duhok}

\section{Received}
15/04/2007
Accepted
17/07/2007

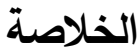

تم تحضير سلسـلة من الفـا-اريليدين هكسانون حلقي (1-4) بواسطة تكاثف كليزن شميدت، بينمـا تم تحضير سلسلة اخرى مـ N-اريليدين بنزايل امين (قواعد شف 5-10) بالاضـافة المولية المتكافئة. ان الاضـافة الحلقة الانيونية 1،3 لقواعد شف على الفـا-راريليدين هكسانون حلقي تحت ظروف قاعدية انتج البايروليدينات المقابلة نوع سبايرو (11-23). لقد تم

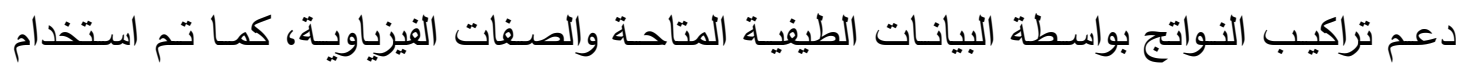
البيانات النظرية لدعم الميكانيكية المقترحة.

\section{ABSTRACT}

A series of $\alpha$-arylidene cyclohexanones (1-4) had been prepared via Claisen-Schmidt condensation, while other series of $\mathrm{N}$-arylidene benzylamines (Schiff bases 5-10) had also been synthesized by equimolar addition. The 1,3-anionic cycloaddition of Schiff bases to $\alpha$-arylidene cyclohexanones under basic conditions afforded the corresponding spiro pyrrolidines (11-23). The structures of the products are supported by valid spectral data and physical properties. Theoretical data had been used to support the suggested mechanism.

\section{INTRODUCTION}

Substituted chiral, nonracemic pyrrolidines are common structural motifs found in many natural and unnatural products that possess interesting and important biological activities, and a great deal of effects has been devoted toward the development of asymmetric methods for their synthesis ${ }^{(1,2)}$. The pyrrolidine ring is found in many natural compounds and may be fused to other rings in a variety of ways as in cocaine, lepadiformine, and (-) - coccinine $^{(3)}$.

We have been interested in developing a pyrrolidine synthesis that would be general enough to use for waking diverse-pyrolidine-containing compounds such as mentioned above $\mathrm{e}^{(4)}$.

Prior to our work, the anionic cycloaddition of 2-azaallyllithiums, a specific type of 2-azaallyl anion, with alkenes was limited to anions bearing two or more aryl groups, chemistry pioneered by Kauffmann ${ }^{(5)}$. The cyclic system of pyrrolidine is found in many important medicines 
which considered as a natural products ${ }^{(6)}$, and it is known that pyrrolidine and its derivatives possess a biological activity. Some substituted pyrrolidines are anti-depressant, anti-psychotic, and analgesic ${ }^{(7)}$, some of which are anti-arythritic and anti-smoking ${ }^{(8)}$, while others are anti-cancer, anti-microbial and anti-hypertensive ${ }^{(9)}$.

Pyrrolidine dithiocarbamate (PDTC) is used as anti-viral agent against virus causes influenza by quenching the gene responsible for this virus $^{(10)}$.

\section{Instrumentation}

\section{EXPERIMENTAL}

1. Boiling points were determined by using an inverted capillary in a Thiele tube using paraffin colourless oil.

2. Melting points were determined by Electrothermal 9000 Digitalseries 1998 apparatus (uncorrected).

3. Ultraviolet spectra were obtained using SPECORD 200 UVVisible double beam Analytikjenta spectrophotometer.

4. Fourier-Transform Infrared (FT-IR) spectra were recorded by using Thermo-Nicolte Fourier-Transform Infrared (FT-IR) spectrophotometer.

5. The theoretical calculations were computed using semi-empirical AM1 module in the CS ChemOffice molecular modeling package. The data obtained from the minimized geometry were used for the theoretical calculations.

H.F = heat of formation

S.E $=$ steric energy

\section{Synthesis}

\section{Preparation of $\alpha$-arylidene cyclohexanone (1-4)}

\section{General procedure ${ }^{(11)}$}

A mixture of (1.1 gm, 0.028 mole) of sodium hydroxide pellets, (10) $\mathrm{ml}$ of water and $(6.0 \mathrm{ml}, 0.1$ mole $)$ of ethanol was magnetically stirred in a $50 \mathrm{ml}$ round-bottomed flask. A fresh distilled cyclohexanone $(2.3 \mathrm{ml}$, 0.022 mole) was poured on the stirred mixture followed by the desired benzaldehyde $(0.022$ mole). A vigorous stirring at room temperature is continued for (3-4) hours, till the mixture become thick, then kept in a refrigerator overnight. The product was filtered by suction and washed several times with water until the filterates were neutral to litmus. The crude $\alpha$-arylidene cyclohexanone is dried in air and recrystallized from ethanol, see Table-1, while the following equation illustrates the reaction:

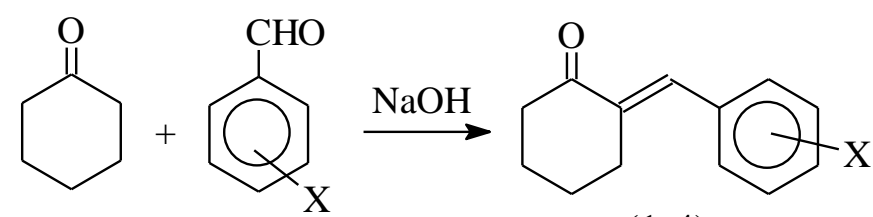

$(1-4)$ 


\section{Preparation of $\mathbf{N}$-arylidene benzylamines (5-10) (Schiff bases) General procedure ${ }^{(12)}$}

A mixture of (1.1 ml, 0.01 mole) of benzylamine and $(0.01 \mathrm{~mole})$ of a desired benzaldehyde is heated in a $100 \mathrm{ml}$ beaker for $(10 \mathrm{~min}$.) at 100 ${ }^{\circ} \mathrm{C}$ after the addition of about (10) ml n-butanol. The precipitated Schiff base was filtered after cooling the mixture and recrystallized from ethanol, see Table-2, the following equation illustrate the formation of the Schiff bases.

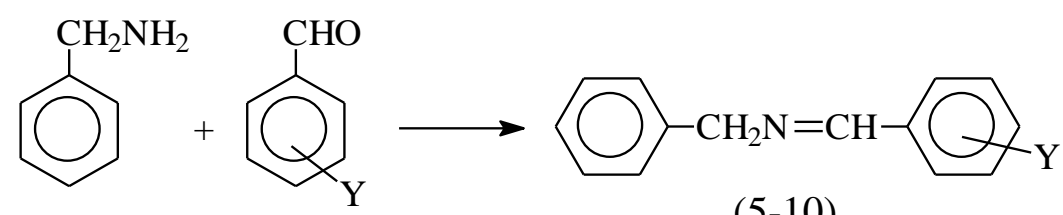

\section{1,3-Anionic cycloaddition of $\mathrm{N}$-arylidene benzylamines to $\alpha$-arylidene cyclohexanone (11-23) \\ General procedure ${ }^{(13)}$}

A mixture of $(0.01$ mole $)$ of $\alpha$-arylidene cyclohexanone and $(0.01$ mole) of Schiff base was stirred in the presence of $(5 \mathrm{ml})$ of $50 \%$ ethanolic sodium hydroxide solution and $(10 \mathrm{ml})$ of dimethyl sulfoxide, stirring at room temperature was continued for (4) hours, then the mixture was allowed to stand overnight. A $(100 \mathrm{ml})$ of water was added to the mixture and separated precipitates were filtered and washed with water till the filtrate seems clear and being neutral. The solid products were dried and recrystallized from methanol-ethyl acetate, Table-3 illustrates the physical and spectral data of the spiro products which had been afforded according to the following equation:

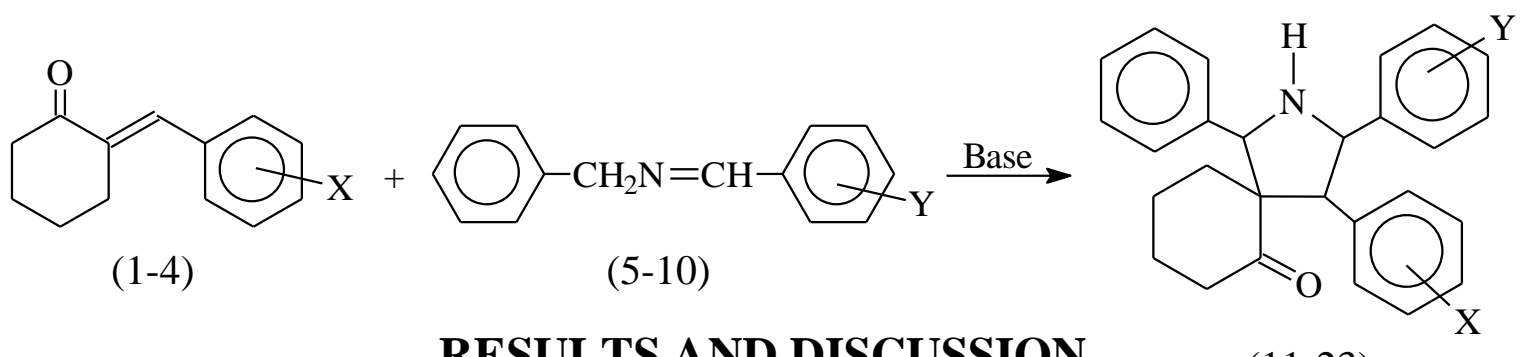

\section{RESULTS AND DISCUSSION}

(11-23)

Claisen-Schimdt condensation had been used to afford the four $\alpha$ arylidene cyclohexanones (1-4) to be used as a starting materials to get the spiro products after their reaction with the synthesized $\mathrm{N}$-arylidene benzylamines (5-10) through 1,3-anionic cycloaddition (Scheme 1). 

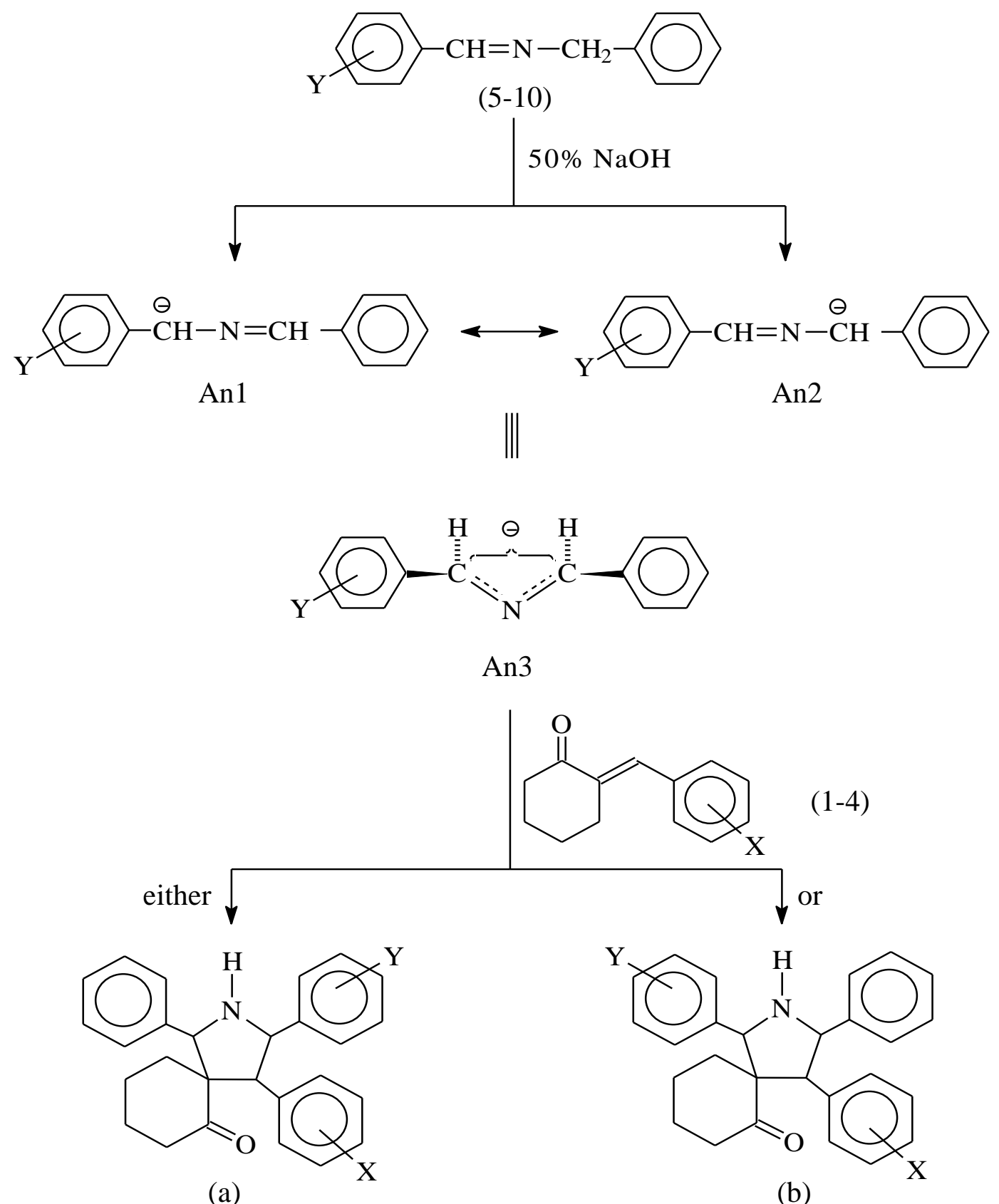

(a)

(b)

Scheme (1): 1,3-Anionic cycloaddition of Schiff bases (5-10) to $\alpha$-arylidene cyclohexanone (1-4)

The UV spectra ${ }^{(14)}$ for (1-4) reflect a range of (354-368) nm, that it quite correlated with the published values of these compounds ${ }^{(15)}\left(\mathrm{n} \rightarrow \pi^{*}\right.$ bands for $\alpha, \beta$-unsaturated ketones).

The IR spectra ${ }^{(16)}$, showed a range of stretching vibrations of (1699$1653) \mathrm{cm}^{-1}$ related to carbonyl group and a range of (1610-1594) $\mathrm{cm}^{-1}$ which concerns the carbon-nitrogen double bond, the stretching vibration at (768) $\mathrm{cm}^{-1}$ is for carbon-chloride bond of compound number (2), finally the vibrations at $(1024) \mathrm{cm}^{-1}$ and (1027) $\mathrm{cm}^{-1}$ are related to the ethereal C-O-C stretching of compound (3) and (4) respectively(Table-1). 
Table (1): Some physical properties and spectral data for $\alpha$-arylidene cyclohexanones (1-4)

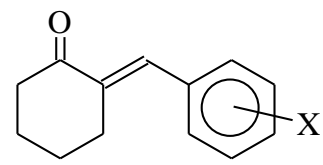

\begin{tabular}{|c|c|c|c|c|c|c|c|c|c|c|c|}
\hline \multirow{2}{*}{$\begin{array}{l}\text { Comp. } \\
\text { No. }\end{array}$} & \multirow[b]{2}{*}{ Name } & \multirow[b]{2}{*}{ X } & \multirow{2}{*}{$\begin{array}{l}\text { M.Wt. } \\
(\mathrm{g} / \mathrm{mol})\end{array}$} & \multirow{2}{*}{$\begin{array}{l}\text { Molecular } \\
\text { formula }\end{array}$} & \multirow{2}{*}{$\begin{array}{l}\text { m.p. } \\
\left({ }^{\circ} \mathrm{C}\right)\end{array}$} & \multirow{2}{*}{$\begin{array}{c}\text { Yield } \\
(\%)\end{array}$} & \multirow[b]{2}{*}{ Colour } & \multicolumn{3}{|c|}{ I.R $(\mathrm{KBr}) \vee \mathrm{cm}^{-1}$} & \multirow{2}{*}{$\begin{array}{c}\mathrm{U} . \mathrm{V} \\
\left(\mathrm{CH}_{3} \mathrm{Cl}\right) \\
\lambda_{\max }(\mathrm{nm})\end{array}$} \\
\hline & & & & & & & & $\mathrm{C}=\mathrm{O}$ & $\mathrm{C}=\mathrm{C}$ & Others & \\
\hline 1 & $\begin{array}{l}\alpha \text {-Benzylidene } \\
\text { cyclohexanone }\end{array}$ & $\mathrm{H}$ & 186 & $\mathrm{C}_{13} \mathrm{H}_{14} \mathrm{O}$ & $102-104$ & 85 & Yellow & 1698 & 1606 & - & 354 \\
\hline 2 & 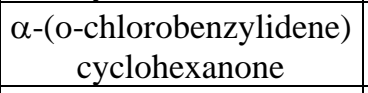 & $\mathrm{o}-\mathrm{Cl}$ & 220 & $\mathrm{C}_{13} \mathrm{H}_{13} \mathrm{OCl}$ & $68-70$ & 85 & Yellow & 1699 & 1610 & $\begin{array}{c}768 \\
\mathrm{C}-\mathrm{Cl} \\
\end{array}$ & 356 \\
\hline 3 & $\begin{array}{c}\alpha-(\mathrm{p}- \\
\text { Methoxybenzylidene) } \\
\text { cyclohexanone }\end{array}$ & $\mathrm{p}-\mathrm{OMe}$ & 216 & $\mathrm{C}_{14} \mathrm{H}_{16} \mathrm{O}_{2}$ & $145-147$ & 90 & Yellow & 1653 & 1594 & $\begin{array}{c}1024 \\
\mathrm{C}-\mathrm{O}-\mathrm{C}\end{array}$ & 368 \\
\hline 4 & $\begin{array}{c}\alpha-(2,4- \\
\text { Dimethoxybenzylidene) } \\
\text { cyclohexanone }\end{array}$ & $\begin{array}{c}2,4- \\
\mathrm{DiMeO}\end{array}$ & 234 & $\mathrm{C}_{15} \mathrm{H}_{18} \mathrm{O}_{3}$ & $150-152$ & 90 & Yellow & - & 1601 & $\begin{array}{c}1027 \\
\text { C-O-C }\end{array}$ & 368 \\
\hline
\end{tabular}

The other starting materials ( $\mathrm{N}$-arylidene benzyl amines 5-10) had been confirmed structurally according to physical and spectral data (Table-2). The UV and IR spectra were in a good agreement with the literature ${ }^{(14,15,17)}$. The UV spectra showed a range of (301-339) $\mathrm{nm}$ that belongs to such compounds (Schiff bases with $\mathrm{C}=\mathrm{N}$ bonds).

Table (2): Some physical properties and spectral data for $\alpha$-arylidene benzylamine (5-10) (Schiff bases)

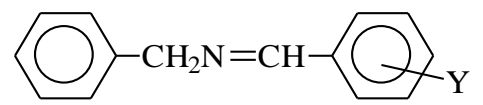

\begin{tabular}{|c|c|c|c|c|c|c|c|c|}
\hline \multirow{2}{*}{$\begin{array}{l}\text { Comp. } \\
\text { No. }\end{array}$} & \multirow[b]{2}{*}{ Name } & \multirow[b]{2}{*}{$\mathrm{Y}$} & \multirow{2}{*}{$\begin{array}{l}\text { m.p. or } \\
\text { b.p. } \\
\left({ }^{\circ} \mathrm{C}\right)\end{array}$} & \multirow{2}{*}{$\begin{array}{c}\text { Yield } \\
(\%)\end{array}$} & \multicolumn{3}{|c|}{ I.R $(\mathrm{KBr}) \vee \mathrm{cm}^{-1}$} & \multirow{2}{*}{$\begin{array}{c}\mathrm{U} . \mathrm{V} \\
\left(\mathrm{CH}_{3} \mathrm{Cl}\right) \\
\lambda_{\max }(\mathrm{nm})\end{array}$} \\
\hline & & & & & $\mathrm{C}=\mathrm{N}$ & $\begin{array}{c}\mathrm{NO}_{2} \\
\text { (asy/sym) }\end{array}$ & Others & \\
\hline 5 & $\begin{array}{c}\mathrm{N}-(\mathrm{m}-\mathrm{Nitrobenzylidene}) \\
\text { benzylamine }\end{array}$ & $\mathrm{m}-\mathrm{NO}_{2}$ & $59-62$ & 80 & 1670 & $\begin{array}{l}1500 \\
1318 \\
\end{array}$ & - & 301 \\
\hline 6 & $\begin{array}{c}\mathrm{N}-(\mathrm{p}-\text { Nitrobenzylidene }) \\
\text { benzylamine }\end{array}$ & $\mathrm{p}-\mathrm{NO}_{2}$ & $50-52$ & 60 & 1675 & $\begin{array}{l}1500 \\
1340\end{array}$ & - & 338 \\
\hline 7 & $\begin{array}{l}\mathrm{N}-(\mathrm{o}-\text { Chlorobenzylidene }) \\
\text { benzylamine }\end{array}$ & $\mathrm{o}-\mathrm{Cl}$ & $139-140$ & 85 & 1665 & - & $\begin{array}{l}705 \\
\mathrm{C}-\mathrm{Cl}\end{array}$ & 336 \\
\hline 8 & $\begin{array}{c}\mathrm{N}-(\mathrm{p}-\text { Bromobenzylidene }) \\
\text { benzylamine }\end{array}$ & $\mathrm{p}-\mathrm{Br}$ & $39-41$ & 85 & 1668 & - & $\begin{array}{c}700 \\
\mathrm{C}-\mathrm{Br}\end{array}$ & 336 \\
\hline 9 & $\begin{array}{l}\text { N-(p-Methoxybenzylidene) } \\
\text { benzylamine }\end{array}$ & $\mathrm{p}-\mathrm{OMe}$ & 202-204 & 75 & 1665 & - & $\begin{array}{c}1145 \\
\mathrm{C}-\mathrm{O}-\mathrm{C}\end{array}$ & 290 \\
\hline 10 & $\begin{array}{c}\mathrm{N}-(2,4-\text { Dimethoxybenzylidene }) \\
\text { benzylamine }\end{array}$ & $\begin{array}{c}2,4- \\
\mathrm{DiMeO}\end{array}$ & $\begin{array}{c}125-126 \\
\text { (liquid) }\end{array}$ & 60 & 1645 & - & $\begin{array}{c}1140 \\
\mathrm{C}-\mathrm{O}-\mathrm{C}\end{array}$ & 339 \\
\hline
\end{tabular}


The afforded pyrrolidines (11-23) and their structures were supported using physical properties and valid spectral data (Table-3). The UV spectra ${ }^{(18)}$ showed a rang of (324-368) $\mathrm{nm}$ which is related to such cycles, that is to say that the presence of three phenyl rings with some different substituents causes the absorption at such $\lambda_{\max }$ in addition to the carbonyl group and the pyrrolidine ring. The most effective transition is the $\mathrm{n} \rightarrow \pi^{*}$ which is highly affected by the solvent.

The IR spectra manifested a range of (1679-1637) $\mathrm{cm}^{-1}$ attributed to the carbonyl stretching vibration ${ }^{(15)}$, while a range of $(3448-3443) \mathrm{cm}^{-1}$ is related to $\mathrm{NH}$ stretching vibration ${ }^{(19)}$, the other $\mathrm{C}-\mathrm{O}-\mathrm{C}$ (ethereal) vibration $^{(20)}$ seemed at the range of (1266-1248) $\mathrm{cm}^{-1}$ for asymmetric stretching and a range of (1034-1025) $\mathrm{cm}^{-1}$ for the symmetrical vibration, finally, the carbon-halogen stretching vibrations denoted at a range of (755-751) $\mathrm{cm}^{-1}$ for $\mathrm{C}-\mathrm{Cl}$ and a range of $(701-582) \mathrm{cm}^{-1}$ for $\mathrm{C}-\mathrm{Br}$. 
Table (3): Some physical properties and spectral data for the spiro pyrrolidines (11-23)

\begin{tabular}{|c|c|c|c|c|c|c|c|c|c|c|}
\hline \multirow{2}{*}{$\begin{array}{l}\text { Comp. } \\
\text { No. }\end{array}$} & \multirow{2}{*}{ Name } & \multirow{2}{*}{$\mathrm{X}$} & \multirow{2}{*}{$\mathrm{Y}$} & \multirow{2}{*}{$\begin{array}{l}\text { m.p. } \\
\left({ }^{\circ} \mathrm{C}\right)\end{array}$} & \multirow{2}{*}{$\begin{array}{c}\text { Yield } \\
(\%)\end{array}$} & \multirow{2}{*}{ Colour } & \multicolumn{3}{|c|}{ I.R $(\mathrm{KBr}) \vee \mathrm{cm}^{-1}$} & \multirow{2}{*}{$\begin{array}{c}\mathrm{U} . \mathrm{V}\left(\mathrm{CH}_{3} \mathrm{Cl}\right) \\
\lambda_{\max }(\mathrm{nm})\end{array}$} \\
\hline & & & & & & & $\mathrm{C}=\mathrm{O}$ & $\mathrm{N}-\mathrm{H}$ & Others & \\
\hline 11 & 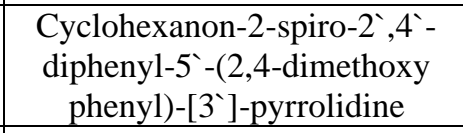 & $\mathrm{H}$ & $\begin{array}{c}2,4- \\
\mathrm{DiMeO}\end{array}$ & $148-150$ & 40 & Brown & 1669 & 3444 & \begin{tabular}{|c|}
1028 \\
1262 \\
$\mathrm{C}-\mathrm{O}-\mathrm{C}$ \\
\end{tabular} & 368 \\
\hline 12 & $\begin{array}{c}\text { Cyclohexanon-2-spiro-2`- } \\
\text { phenyl-4`-(o-chlorophenyl)-5 - } \\
\text { (2,4-dimethoxy phenyl)-[3`]- } \\
\text { pyrrolidine } \\
\end{array}$ & $\mathrm{o}-\mathrm{Cl}$ & $\begin{array}{c}2,4- \\
\mathrm{DiMeO}\end{array}$ & $100-102$ & 40 & Brown & 1678 & 3448 & \begin{tabular}{|c|}
1034 \\
1266 \\
$\mathrm{C}-\mathrm{O}-\mathrm{C}$ \\
$751 \mathrm{C}-\mathrm{Cl}$ \\
\end{tabular} & 338 \\
\hline 13 & $\begin{array}{l}\text { Cyclohexanon-2-spiro-2`,4`- } \\
\text { diphenyl-5`-(p-nitrophenyl)- } \\
\text { [3`]-pyrrolidine }\end{array}$ & $\mathrm{H}$ & $\mathrm{p}-\mathrm{NO}_{2}$ & $198-200$ & 35 & Red & 1641 & 3445 & $\begin{array}{c}1341 \\
\mathrm{C}-\mathrm{NO}_{2}\end{array}$ & 360 \\
\hline 14 & $\begin{array}{c}\text { Cyclohexanon-2-spiro-2`- } \\
\text { phenyl-4`-(p-methoxy phenyl)- } \\
\text { 5'-(2,4-dimethoxy phenyl)- } \\
\text { [3`]-pyrrolidine }\end{array}$ & $\mathrm{p}-\mathrm{OMe}$ & $\begin{array}{c}2,4- \\
\mathrm{DiMeO}\end{array}$ & $220-222$ & 40 & Yellow & 1676 & 3444 & $\begin{array}{c}1026 \\
1251 \\
\mathrm{C}-\mathrm{O}-\mathrm{C}\end{array}$ & 368 \\
\hline 15 & $\begin{array}{c}\text { Cyclohexanon-2-spiro-2`- } \\
\text { phenyl-4`-(p-methoxy phenyl)- } \\
5^{`} \text {-(m-nitrophenyl)-[3`]- } \\
\text { pyrrolidine }\end{array}$ & $\mathrm{p}-\mathrm{OMe}$ & $\mathrm{m}-\mathrm{NO}_{2}$ & $120-122$ & 40 & $\begin{array}{c}\text { Dark } \\
\text { brown }\end{array}$ & 1679 & 3443 & $\begin{array}{c}1027 \\
1250 \\
\mathrm{C}-\mathrm{O}-\mathrm{C} \\
1347 \mathrm{C}- \\
\mathrm{NO}_{2} \\
\end{array}$ & 368 \\
\hline 16 & $\begin{array}{c}\text { Cyclohexanon-2-spiro-2`- } \\
\text { phenyl-4'-(2,5-dimethoxy } \\
\text { phenyl)-5'-(2,4-dimethoxy } \\
\text { phenyl)-[3’]-pyrrolidine } \\
\end{array}$ & $\begin{array}{c}2,5- \\
\mathrm{DiMeO}\end{array}$ & $\begin{array}{c}2,4- \\
\mathrm{DiMeO}\end{array}$ & $220-224$ & 30 & Yellow & 1661 & 3448 & $\begin{array}{c}1028 \\
1264 \\
\mathrm{C}-\mathrm{O}-\mathrm{C}\end{array}$ & 368 \\
\hline 17 & \begin{tabular}{|c|} 
Cyclohexanon-2-spiro-2`- \\
phenyl-4-(2,5-dimethoxy \\
phenyl)-5-(p-bromophenyl)- \\
[3`]-pyrrolidine \\
\end{tabular} & $\begin{array}{c}2,5- \\
\mathrm{DiMeO}\end{array}$ & $\mathrm{p}-\mathrm{Br}$ & $118-120$ & 50 & $\begin{array}{l}\text { Pale } \\
\text { yellow }\end{array}$ & 1668 & 3443 & \begin{tabular}{|c|}
1032 \\
1251 \\
$\mathrm{C}-\mathrm{O}-\mathrm{C}$ \\
$701 \mathrm{C}-\mathrm{Br}$ \\
\end{tabular} & 366 \\
\hline 18 & $\begin{array}{c}\text { Cyclohexanon-2-spiro-2`- } \\
\text { phenyl-4`-(p-methoxy phenyl)- } \\
5^{5} \text {-(b-bromophenyl)-[3`]- } \\
\text { pyrrolidine } \\
\end{array}$ & $\mathrm{p}-\mathrm{MeO}$ & $\mathrm{p}-\mathrm{Br}$ & $108-110$ & 70 & yellow & 1666 & 3443 & \begin{tabular}{|c|}
1033 \\
1263 \\
$\mathrm{C}-\mathrm{O}-\mathrm{C}$ \\
$582 \mathrm{C}-\mathrm{Br}$ \\
\end{tabular} & 368 \\
\hline 19 & $\begin{array}{c}\text { Cyclohexanon-2-spiro-2`- } \\
\text { phenyl-4`,5-di(p-methoxy } \\
\text { phenyl)-[3']-pyrrolidine }\end{array}$ & $\mathrm{p}-\mathrm{MeO}$ & $\mathrm{p}-\mathrm{MeO}$ & $92-94$ & 85 & White & 1640 & 3443 & \begin{tabular}{|c|}
1034 \\
1248 \\
C-O-C \\
\end{tabular} & 358 \\
\hline 20 & $\begin{array}{c}\text { Cyclohexanon-2-spiro-2 - } \\
\text { phenyl-4-(2,5-dimethoxy } \\
\text { phenyl)-5'-(p-methoxy } \\
\text { phenyl)-[3']-pyrrolidine }\end{array}$ & $\begin{array}{c}2,5- \\
\mathrm{DiMeO}\end{array}$ & $\mathrm{p}-\mathrm{MeO}$ & $98-100$ & 80 & White & 1641 & 3444 & $\begin{array}{c}1033 \\
1249 \\
\text { C-O-C }\end{array}$ & 336 \\
\hline 21 & $\begin{array}{c}\text { Cyclohexanon-2-spiro-2`- } \\
\text { phenyl-4^-(o-chlorophenyl)-5 - } \\
\text { (p-methoxy phenyl)-[3`]- } \\
\text { pyrrolidine }\end{array}$ & $\mathrm{o}-\mathrm{Cl}$ & $\mathrm{p}-\mathrm{MeO}$ & 90-92 & 80 & $\begin{array}{l}\text { Yellowish } \\
\text { white }\end{array}$ & 1640 & 3444 & \begin{tabular}{|c|}
1033 \\
1248 \\
$\mathrm{C}-\mathrm{O}-\mathrm{C}$ \\
$751 \mathrm{C}-\mathrm{Cl}$ \\
\end{tabular} & 324 \\
\hline 22 & $\begin{array}{c}\text { Cyclohexanon-2-spiro-2`- } \\
\text { phenyl-4^-(o-chlorophenyl)-5 - } \\
\text { (b-bromophenyl)-[3`]- } \\
\text { pyrrolidine }\end{array}$ & $\mathrm{o}-\mathrm{Cl}$ & $\mathrm{p}-\mathrm{Br}$ & $122-124$ & 90 & White & 1637 & 3444 & $\begin{array}{c}7003 \\
\mathrm{C}-\mathrm{Br} \\
752 \mathrm{C}-\mathrm{Cl}\end{array}$ & 342 \\
\hline 23 & $\begin{array}{c}\text { Cyclohexanon-2-spiro-2`- } \\
\text { phenyl-4`-(p-methoxy phenyl)- } \\
5^{`} \text {-(o-chlorophenyl)-[3`]- } \\
\text { pyrrolidine }\end{array}$ & $\mathrm{p}-\mathrm{MeO}$ & $\mathrm{o}-\mathrm{Cl}$ & $152-154$ & 30 & Yellow & 1657 & 3444 & \begin{tabular}{|c|}
1025 \\
1250 \\
$\mathrm{C}-\mathrm{O}-\mathrm{C}$ \\
$755 \mathrm{C}-\mathrm{Cl}$ \\
\end{tabular} & 368 \\
\hline
\end{tabular}


The 1,3-cycloaddition reaction of Schiff bases (5-10) to $\alpha$-arylidene cyclohexanones (1-4) is passed through the following suggested mechanism $^{(21)}$ (Scheme 1).

It is obvious that the strong base $(\mathrm{NaOH})$ may abstracts an acidic proton from the Schiff base to afford the anion An1 which in turn resonate to give the anion An2, both An1 and An2 can be represented by the equivalent hybrid An3.

The addition of one mole of $\mathrm{N}$-arylidene cyclohexanone to An3 may lead to the production of the isomer (a) or the isomer (b). In the light of the values of the heat of formation (H.F) and the steric energy (S.E) (Table-4), it is concluded that (a) may be predominate.

Table (4): Heat of formation (H.F) and steric energy (S.E) of final products (11-23)

\begin{tabular}{|c|c|c|}
\hline Comp. No. & H.F (Kcal/mole) & S.E (Kcal/mole) \\
\hline $11 \mathrm{a}$ & -26.72764 & 37.67815 \\
\hline $11 \mathrm{~b}$ & -26.05804 & 37.32981 \\
\hline $12 \mathrm{a}$ & -27.03265 & 45.94420 \\
\hline $12 \mathrm{~b}$ & -27.00822 & 45.26365 \\
\hline $13 \mathrm{a}$ & 50.65342 & 37.53821 \\
\hline $13 \mathrm{~b}$ & 49.53609 & 39.65823 \\
\hline $14 \mathrm{a}$ & -64.74389 & 45.70163 \\
\hline $14 \mathrm{~b}$ & -65.21534 & 48.71519 \\
\hline $15 \mathrm{a}$ & 14.49786 & 37.95341 \\
\hline $15 \mathrm{~b}$ & 11.49595 & 38.75468 \\
\hline $16 \mathrm{a}$ & -97.77710 & 55.75074 \\
\hline $16 \mathrm{~b}$ & -97.79879 & 57.94244 \\
\hline $17 \mathrm{a}$ & -20.22194 & 36.12594 \\
\hline $17 \mathrm{~b}$ & -19.68219 & 36.65040 \\
\hline $18 \mathrm{a}$ & 14.84119 & 29.17400 \\
\hline $18 \mathrm{~b}$ & 14.06340 & 29.33428 \\
\hline $19 \mathrm{a}$ & -22.79252 & 37.39347 \\
\hline $19 \mathrm{~b}$ & -27.89615 & 38.50438 \\
\hline $20 \mathrm{a}$ & -62.09895 & 46.16057 \\
\hline $20 \mathrm{~b}$ & -62.31352 & 46.74833 \\
\hline $21 \mathrm{a}$ & 9.35865 & 33.20892 \\
\hline $21 \mathrm{~b}$ & 9.73087 & 34.22443 \\
\hline $22 \mathrm{a}$ & 50.56105 & 25.24840 \\
\hline $22 \mathrm{~b}$ & 50.00597 & 25.07982 \\
\hline $23 \mathrm{a}$ & 4.37232 & 30.00714 \\
\hline $23 \mathrm{~b}$ & 4.49245 & 30.67931 \\
\hline & & \\
\hline
\end{tabular}

Prior to this work ${ }^{(22)}$, it was found that fused pyrrolidines had been prepared by the 1,3-anionic cycloaddition of a series of Schiff bases to 1,4-benzoquinone and the reaction was controlled by the type of the substituent on the Schiff base and it was found that electron-donating 
groups need less energy to afford the final product compared with the electron-withdrawing groups. In the present work it is found that both $\alpha$-arylidene cyclohexanones (1-4) and Schiff bases (5-10) control the rate of the reaction and H.F (-97) Kcal/mole for dimethoxy derivatives is the lowest value compared with H.F (50.561 and 50.563) Kcal/mole for the nitro derivative and the chlorobromo derivative respectively, which means that electron-donation enhances the rate and electron-withdrawal inhibits the rate of the reaction.

\section{REFERENCES}

1. O'Hagan D., Nat. Prod. Rep., 17: 435-446 (2000).

2. Ling R., Ekhato I.V., Rubin J.R., Wustrow D.J., Tetrahedron, 57: 6579-6588 (2001).

3. Pearson W.H., Pure Appl. Chem., 74(8): 1339-1347 (2002).

4. Al-Hamdany A.J., Mustapha A.M., Abdul-Rahman M.S., J. Edu. Sci., 19(3): 33-42 (2007).

5. T. Kauffmann, Angew. Chem., Int. Ed. Engl., 13: 627-639 (1974).

6. Liotta's L., "Louis Liotta's Research Interest", American Chemical Society Inc., p. 1 (2003).

7. Abbott, J. Med. Chem., 30: 1433-1454 (1987).

8. Glaxo, J. Med. Chem., 39: 4478-4482 (1996).

9. Vicker N., et al., J. Med. Chem., 45: 721 (2002).

10.Cox N.J., Kawoka, "Influenza in Topely", $9^{\text {th }}$ Ed., Arnold, London, UK, 385-433 (1998).

11. Vogel I., A., "Practical Organic Chemistry", $2^{\text {nd }}$ Ed., Longmans, pp. 676-681 (1951).

12.Vogel II, A., "Practical Organic Chemistry", $2^{\text {nd }}$ Ed., Longmans, 625 (1951).

13.Popandova-Yambolieva K. and Ivanov C., Chemica Scripta., 29: 269-271 (1989).

14.Williams D.H. and Fleming I., "Spectroscopic Methods in Organic Chemistry", $2^{\text {nd }}$ Ed., McGraw-Hill Book Company (UK), 29 (1973).

15.Toda F., Tanaka K. and Koki Hamai, Perkin Transactions 1: 32073209 (1990).

16.Ref. 14, p. 57.

17.Ref. 14, p. 60.

18.Ref. 14, p. 31.

19.Ref. 14 , p. 51

20.Ref. 14, p. 65.

21.Al-Hamdany A.J., Ph.D. Thesis, Mosul University, 152-154 (2002) (In Arabic).

22.Ref. 4, p. 38. 